МАТЕМАТИКА

MATHEMATICS

https://doi.org/10.15407/dopovidi2021.04.009

УДК 512.812 .4

Д.Р. Попович, https://orcid.org/0000-0001-5563-966X

Інститут математики НАН України, Київ

E-mail: deviuss@gmail.com

\title{
Прапори підалгебр у контрактованих алгебрах Лі
}

Представлено членом-кореспондентом НАН украӥни А.Г. Нікітіним

Доведено теорему, що описує поведінку прапорів підалгебр при контракціях алгебр Лі та яку можна розглядати як новий критерій неіснування контракцій. Отримано також ослаблений аналог иієі теореми для прапорів підпросторів. За ї допомогою показано неіснування контракцій для низки пар шестивимірних нільпотентних дійсних алгебр Лі, для яких не пращюють раніме відомі критерії.

Ключові слова: контракції алгебр Лі, прапори підалгебр, прапори підпросторів у алгебрах Лі, шестивимірні нільпотентні алгебри Лі.

Контракції є різновидом граничних переходів, що формально описують зв’язки між алгебраїчними структурами, зокрема тими, що лежать в основі фізичних теорій. Контракції розглядають для алгебр над дійсним або комплексним полем. Для більш загального випадку довільних алгебраїчно замкнених полів існує поняття вироджень алгебр Лі [1-3]. Незважаючи на інтенсивні дослідження, повного розуміння, які властивості зберігаються при контракціях, дотепер немає.

У цій статті вивчено поведінку прапорів підалгебр і підпросторів при контракціях алгебр Лі. Отримані властивості можна використати як нові критерії неіснування контракцій у парах алгебр. Цю можливість продемонстровано доведенням неіснування контракцій для низки пар шестивимірних нільпотентних дійсних алгебр Лi, для яких не працюють відомі в літературі критерії, оскільки в парах відповідних комплексифікацій контракції існують.

Розглянемо $n$-вимірний $(n<\infty)$ векторний простір $V$ над полем $\mathbb{F}=\mathbb{C}$ або $\mathbb{F}=\mathbb{R}$ та визначену на $V$ бінарну операцію $\mu=[\cdot, \cdot]: V \times V \rightarrow V$. Якщо ця операція є білінійною, кососиметричною та задовольняє тотожність Якобі, то простір 3 нею називають алгеброю Лі $\mathfrak{g}=(V, \mu)$. У фіксованому базисі $\left(e_{1}, \ldots, e_{n}\right)$ простору $V$ алгебру $\mathfrak{g}$ можна визначити через дужку Лі базисних елементів: $\left[e_{i}, e_{j}\right]=c_{i j}^{k} e_{k}$, де $c_{i j}^{k}$ називають структурними сталими алгебри $\mathfrak{g}$ у базисі $\left(e_{1}, \ldots, e_{n}\right)$. Тут і далі індекси $i, j, k$ пробігають від 1 до $n$, за повтореними індексами йде підсумовування. Многовид тензорів структурних сталих алгебр Лі на просторі $V$ - це множина

Ци т у в ан н я: Попович Д.Р. Прапори підалгебр у контрактованих алгебрах Лі. Допов. Наи. акад. наук Укp. 2021. № 4. С. 9-17. https://doi.org/10.15407/dopovidi2021.04.009 


$$
\mathcal{C}_{n}=\left\{\left(c_{i j}^{k}\right) \in \mathbb{F}^{n^{3}} \mid c_{i j}^{k}+c_{j i}^{k}=0, c_{i j}^{i^{\prime}} c_{i^{\prime} k}^{k^{\prime}}+c_{k i}^{i^{\prime}} c_{i^{\prime} j}^{k^{\prime}}+c_{j k}^{i^{\prime}} c_{i^{\prime} i}^{k^{\prime}}=0\right\}
$$

Для матричнозначної функції $U:(0,1] \rightarrow \mathrm{GL}(n), \varepsilon \mapsto U_{\varepsilon}$, і кожного $\varepsilon \in(0,1]$ визначимо перетворену дужку Лі $\mu_{\varepsilon}=\mu \circ U_{\varepsilon}=[\cdot, \cdot]_{\varepsilon}: V \times V \rightarrow V$ згідно $3 \mu_{\varepsilon}(x, y):=U_{\varepsilon}^{-1} \mu\left(U_{\varepsilon} x, U_{\varepsilon} y\right)$, $x, y \in V$. Якщо для довільних $x, y \in V$ існує границя $\lim _{\varepsilon \rightarrow+0} \mu(x, y)_{\varepsilon}=: \mu_{0}(x, y)$, то $\mu_{0}=:[\cdot, \cdot]_{0}$ $\epsilon$ добре визначеною дужкою Лі. Алгебру Лі $\mathfrak{g}_{0}=\left(V, \mu_{0}\right)$ називають неперервною контракцією (або просто контракцією) алгебри Лi $\mathfrak{g}$. Процедуру $\mathfrak{g} \rightarrow \mathfrak{g}_{0}$, за допомогою якої алгебру Лi $\mathfrak{g}_{0}$ отримують з алгебри $\mathfrak{g}$, також називають контракцією. Параметр $\varepsilon$ і матричнозначну функцію $U_{\varepsilon}$ називають відповідно параметром контракцї та матрищею контракцї. У фіксованому базисі простору $V$ оператор $U_{\varepsilon}$ задають матрицею, а означення контракції можна переписати в термінах структурних сталих. А саме: воно означає, що для всіх значень $i^{\prime}, j^{\prime}$, $k^{\prime}$ існує границя $\lim _{\varepsilon \rightarrow+0} c_{\varepsilon, i j}^{k}=: c_{0, i^{\prime} j^{\prime}}^{k^{\prime}}$ де $c_{\varepsilon, i j}^{k}:=\left(U_{\varepsilon}\right)_{i^{\prime}}^{i}\left(U_{\varepsilon}\right)_{j^{\prime}}^{j}\left(U_{\varepsilon}^{-1}\right)_{k}^{k^{\prime}} c_{i j}^{k}$, а тому $c_{0, i^{\prime} j^{\prime}}^{k^{\prime}}$ складають добре визначений тензор структурних сталих алгебри $\mathfrak{g}_{0}$.

Аналогічно до неперервних контракцій можна визначити послідовні контракції, використовуючи послідовності матриць $\left\{U_{p}, p \in \mathbb{N}\right\} \subset \mathrm{GL}(V)$ замість матричнозначних функцій. Для кожної дужки Лі з послідовності $\left\{\mu_{p}=\mu \circ U_{p}=[\cdot, \cdot]_{p}, p \in \mathbb{N}\right\}$ алгебра Лі $\mathfrak{g}_{p}=\left(V, \mu_{p}\right)$ ізоморфна алгебрі $\mathfrak{g}=(V, \mu)$. Якщо границя

$$
\lim _{p \rightarrow \infty} \mu_{p}(x, y)=\lim _{p \rightarrow \infty} U_{p}^{-1} \mu\left(U_{p} x, U_{p} y\right)=: \mu_{0}(x, y)
$$

існує для довільних $x, y \in V$, то $\mu_{0}$ є добре визначеною дужкою Лі на просторі $V$. Алгебру Лi $\mathfrak{g}_{0}=\left(V, \mu_{0}\right)$ називають послідовною контракцією алгебри Лі $\mathfrak{g}$. У фіксованому базисі кожній алгебрі $\mathfrak{g}_{p}$ відповідає тензор структурних сталих $C_{p}=C \circ U_{p}$ з компонентами $c_{p, i j}^{k}=$ $=\left(U_{p}\right)_{i}^{i^{\prime}}\left(U_{p}\right)_{j}^{j^{\prime}}\left(U_{p}^{-1}\right)_{k^{\prime}}^{k} c_{i^{\prime} j^{\prime}}^{k^{\prime}}$. Існування поточкової границі послідовності дужок Лі $\left\{\mu_{p}, p \in \mathbb{N}\right\}$ еквівалентне існуванню границі $\lim _{p \rightarrow \infty} c_{p, i j}^{k}=: c_{0, i j}^{k}$ для всіх значень $i, j, k$, де $c_{0, i j}^{k} \in$ компонентами тензора структурних сталих $C_{0}$ алгебри Лі $\mathfrak{g}_{0}$. Існування неперервної контракції $\mathfrak{g} \rightarrow \mathfrak{g}_{0}$ для пари алгебр $\left(\mathfrak{g}, \mathfrak{g}_{0}\right)$ еквівалентне існуванню послідовної контракції для цієї пари.

Добре відомою є така лема [4], на якій грунтується доведення основного результату цієї роботи:

Лема 1. Алгебра Лі $\mathfrak{g}$ допускає послідовну контракцію до алгебри Лi $\mathfrak{g}_{0}$ тоді $і$ лише тодi, коли у базисі $\left(e_{1}, \ldots, e_{n}\right)$ базового простору $V$ існують послідовність невироджених нижньтрикутних (або верхньотрикутних) $n \times n$ матрищь $\left\{L_{p}, p \in \mathbb{N}\right\}$ ортогональна (відповідно, унітарна) $n \times n$ матрищя $Q$ у дійсному (відповідно, комплексному) випадку такі, що $C \circ L_{p} \rightarrow C_{0} \circ Q$ при $p \rightarrow \infty$.

Основна теорема та ії наслідки. Структура множин підалгебр та ідеалів алгебр Лі змінюється при контракціях. Водночас деякі їх властивості є стабільними.

Теорема 1. Припустимо, що алгебра Лi $\mathfrak{g}_{0} \in$ (неперервною чи послідовною) контракцією алгебри Лi $\mathfrak{g}, \mathfrak{g} \rightarrow \mathfrak{g}_{0}$, i алгебра $\mathfrak{g}$ містить прапор підалгебр

$$
\{0\}=\mathfrak{s}^{0} \subset \mathfrak{s}^{1} \subset \mathfrak{s}^{2} \subset \cdots \subset \mathfrak{s}^{m} \subset \mathfrak{s}^{m+1}=\mathfrak{g} .
$$

Тоді алгебра $\mathfrak{g}_{0}$ містить прапор підалгебр

$$
\{0\}=\mathfrak{s}_{0}^{0} \subset \mathfrak{s}_{0}^{1} \subset \mathfrak{s}_{0}^{2} \subset \cdots \subset \mathfrak{s}_{0}^{m} \subset \mathfrak{s}_{0}^{m+1}=\mathfrak{g}_{0}
$$


таких, що

$$
\operatorname{dim} \mathfrak{s}_{0}^{a}=\operatorname{dim} \mathfrak{s}^{a} \quad m a \quad \mathfrak{s}^{a} \rightarrow \mathfrak{s}_{0}^{a}, \quad a=1, \ldots, m .
$$

Якщо $\mathfrak{s}^{a} \in$ ідеалом $y \mathfrak{s}^{b}, 1 \leqslant a<b \leqslant m+1$, то $\mathfrak{s}_{0}^{a}$ можна обрати ідеалом $y \mathfrak{s}_{0}^{b}$, причому $\mathfrak{s}^{b} / \mathfrak{s}^{a} \rightarrow \mathfrak{s}_{0}^{b} / \mathfrak{s}_{0}^{a}$.

Більш загально, якщо $\left[\mathfrak{s}^{a}, \mathfrak{s}^{b}\right] \subseteq \mathfrak{s}^{c}$ для деяких $a, b, c \in\{1, \ldots, m\}$, то також $\left[\mathfrak{s}_{0}^{a}, \mathfrak{s}_{0}^{b}\right]_{0} \subseteq \mathfrak{s}_{0}^{c}$. Крім того, $\operatorname{dim}\left[\mathfrak{s}_{0}^{a}, \mathfrak{s}_{0}^{b}\right]_{0} \leqslant \operatorname{dim}\left[\mathfrak{s}^{a}, \mathfrak{s}^{b}\right]$ для будъ-яких $a, b \in\{1, \ldots, m\}$. Аналогічні твердження справедливі для будь-якої композииї комутаторів на довільному розміщенні з повтореннями з підалгебр $\mathfrak{s}^{a}$.

Доведення. Достатньо розглянути випадок послідовних контракцій. Виберемо узгоджений з прапором підалгебр в алгебрі $\mathfrak{g}=(V, \mu)$ базис $\left(e_{1}, \ldots, e_{n}\right)$ простору $V$, тобто $\mathfrak{s}^{a}=\left\langle e_{1}, \ldots, e_{n_{a}}\right\rangle, a=1, \ldots, m$, де $n_{a}:=\operatorname{dim}_{\mathfrak{s}^{a}}$. У цьому базисі комутаційні сталі алгебри $\mathfrak{g}$ задовольняють умову

$$
c_{i j}^{k}=0, \quad \text { якщо } i, j \leqslant n_{a} \text { та } k>n_{a} .
$$

Оскільки алгебра $\mathfrak{g}_{0}=\left(V, \mu_{0}\right)$ є контракцією алгебри $\mathfrak{g}$, то згідно з лемою 1 існують послідовність $\left\{L_{p}, p \in \mathbb{N}\right\}$ невироджених верхньотрикутних $n \times n$ матриць і ортогональна (відповідно, унітарна) $n \times n$ матриця $Q$ у дійсному (відповідно, комплексному) випадку такі, що $\mu_{p}:=\mu \circ L_{p} \rightarrow \mu_{0} \circ Q$ при $p \rightarrow \infty$. У кожній алгебрі з послідовності $\left\{\mathfrak{g}_{p}:=\left(V, \mu_{p}\right), p \in \mathbb{N}\right\}$ маємо прапор

$$
\{0\}=\mathfrak{s}_{p}^{0} \subset \mathfrak{s}_{p}^{1} \subset \mathfrak{s}_{p}^{2} \subset \cdots \subset \mathfrak{s}_{p}^{m} \subset \mathfrak{s}_{p}^{m+1}=\mathfrak{g}_{p}:=\left(V, \mu_{p}\right),
$$

де $\mathfrak{s}_{p}^{a}:=L_{p^{\mathfrak{s}}}{ }^{a}=\left\langle e_{1}, \ldots, e_{n_{a}}\right\rangle, a=1, \ldots, m+1$, а тому $\operatorname{dim} \mathfrak{s}_{p}^{a}=\operatorname{dim} \mathfrak{s}^{a}$ i $\mathfrak{s}_{p}^{a} \simeq \mathfrak{s}^{a}$. Іншими словами, комутаційні сталі алгебр $\mathfrak{g}_{p}$ задовольняють умови

$$
c_{p, i j}^{k}=0, \quad \text { якщо } i, j \leqslant n_{a} \text { та } k>n_{a} .
$$

У границі при $p \rightarrow \infty$ маємо $c_{p, i j}^{k} \rightarrow c_{0, i j}^{k}$, звідки $c_{0, i j}^{k}=0$, якщо $i, j \leqslant n_{a}$ та $k>n_{a}$. Це означає, що $\mathfrak{s}_{0}^{a}=\left\langle e_{1}, \ldots, e_{n_{a}}\right\rangle, a=1, \ldots, m,-$ підалгебри алгебри $\mathfrak{g}_{0}$, які утворюють прапор

$$
\{0\}=\mathfrak{s}_{0}^{0} \subset \mathfrak{s}_{0}^{1} \subset \mathfrak{s}_{0}^{2} \subset \cdots \subset \mathfrak{s}_{0}^{m} \subset \mathfrak{s}_{0}^{m+1}=\mathfrak{g}_{0}, \quad \text { де } \quad \operatorname{dim} \mathfrak{s}_{0}^{a}=\operatorname{dim} \mathfrak{s}^{a}=n_{a} .
$$

Крім того, обмеження $\left(c_{i j}^{k}\right)_{i, j, k=1, \ldots, n}$ тензора структурних сталих алгебри $\mathfrak{g} є$ тензором структурних сталих підалгебри $\mathfrak{s}^{a}$, лінійна оболонка $\left\langle e_{1}, \ldots, e_{n_{a}}\right\rangle$ інваріантна під дією оператора $L_{p}$, а тому обмеження $\left(c_{p, i j}^{k}\right)_{i, j, k=1, \ldots, n}$ тензора структурних сталих алгебри $\mathfrak{g}_{p} \in$ тензором структурних сталих підалгебри $\mathfrak{s}_{p}^{a}$. 3 існування границь $c_{p, i j}^{k} \rightarrow c_{0, i j}^{k}, i, j, k=1, \ldots, n_{a}$, при $p \rightarrow \infty$ випливає, що $\mathfrak{s}^{a} \rightarrow \mathfrak{s}_{0}^{a}$.

Нехай для деяких $a, b \in\{1, \ldots, m+1\}$ підалгебра $\mathfrak{s}^{a}$ є ідеалом підалгебри $\mathfrak{s}^{b}$. Аналогічними використаним вище міркуваннями отримаємо, що підалгебра $\mathfrak{s} p$ є ідеалом підалгебри $\mathfrak{s}_{p}^{b}$. У термінах структурних сталих зазначене еквівалентне умові

$$
c_{i j}^{k}=c_{p, i j}^{k}=0 \text { при } i, j \leqslant n_{b}, \quad k>n_{a} \text { та }\left(i \leqslant n_{a} \text { або } j \leqslant n_{a}\right) .
$$


Перехід до границі дає таку саму умову для структурних сталих $c_{0, i j}^{k}$, тобто підалгебра $\mathfrak{s}_{0}^{a}-$ ідеал підалгебри $\mathfrak{s}_{0}^{b}$. Для кожного $p \in \mathbb{N}$ фактор-алгебра $\mathfrak{s}_{p}^{b} / \mathfrak{s}_{p}^{a}$ ізоморфна фактор-алгебрі $\mathfrak{s}^{b} / \mathfrak{s}^{a}$. Структурні сталі $c_{p, i j}^{k}, i, j, k=n_{a}+1, \ldots, n_{b}$, алгебри $\mathfrak{g}_{p}$ утворюють повну множину структурних сталих алгебри $\mathfrak{s}_{p}^{b} / \mathfrak{s}_{p}^{a}$. Аналогічно, підмножина структурних сталих $\left\{c_{0, i j}^{k}, i, j, k=n_{a}+1, \ldots, n_{b}\right\}$ алгебри $\mathfrak{g}_{0} \in$ повною множиною структурних сталих факторалгебри $\mathfrak{s}_{0}^{b} / \mathfrak{s}_{0}^{a}$. Обмеження умови $c_{p, i j}^{k} \rightarrow c_{0, i j}^{k}, \quad p \rightarrow \infty$, що виконується для всіх $i, j, k=1, \ldots, n$, на значення $i, j, k=n_{a}+1, \ldots, n_{b}$ вказує на існування контракції $\mathfrak{s}^{b} / \mathfrak{s}^{a} \rightarrow \mathfrak{s}_{0}^{b} / \mathfrak{s}_{0}^{a}$ між фактор-алгебрами.

Доведемо твердження щодо комутаторів підалгебр.

З умови $\left[\mathfrak{s}^{a}, \mathfrak{s}^{b}\right] \subseteq \mathfrak{s}^{c}$ випливає, що $\left[\mathfrak{s}_{p}^{a}, \mathfrak{s}_{p}^{b}\right]_{p} \subseteq \mathfrak{s}_{p}^{c}$ для кожного $p \in \mathbb{N}$. У термінах структурних сталих це означає умову

$$
c_{i j}^{k}=c_{p, i j}^{k}=0 \text { при }\left(i \leqslant n_{a}, j \leqslant n_{b} \text { або } i \leqslant n_{b}, j \leqslant n_{a}\right) \text { та } k>n_{c},
$$

що в границі дає таку саму умову для структурних сталих $c_{0, i j}^{k}$, звідки $\left[\mathfrak{s}_{0}^{a}, \mathfrak{s}_{0}^{b}\right]_{0} \subseteq \mathfrak{s}_{0}^{c}$.

Зафіксуємо ранжування пар $(i, j) \in\left\{1, \ldots, n_{a}\right\} \times\left\{1, \ldots, n_{b}\right\}$. Розмірності комутаторів $\left[\mathfrak{s}^{a}, \mathfrak{s}^{b}\right]$ $\mathrm{i}\left[\mathfrak{s}_{p}^{a}, \mathfrak{s}_{p}^{b}\right]_{p}$ збігаються між собою, а також з рангами допоміжних матриць $A \mathrm{i} A_{p}$, складених відповідно зі стовпчиків $\left(c_{i j}^{k}\right)_{k=1, \ldots, n}$ i $\left(c_{p, i j}^{k}\right)_{k=1, \ldots, n}$, які занумеровані парами $(i, j)$ у зафіксованому ранжуванні. Аналогічно, розмірність комутатора $\left[\mathfrak{s}_{0}^{a}, \mathfrak{s}_{0}^{b}\right]_{0}$ збігається з рангом допоміжної матриці $A_{0}$, складеної зі стовпчиків $\left(c_{0, i j}^{k}\right)_{k=1, \ldots, n} 3$ такою самою нумерацією, як вище. Очевидно, що $A_{p} \rightarrow A_{0}$ при $p \rightarrow \infty$. Ранг матриць не збільшується при граничному переході. Отже, $\operatorname{dim}\left[\mathfrak{s}_{0}^{a}, \mathfrak{s}_{0}^{b}\right]_{0} \leqslant \operatorname{dim}\left[\mathfrak{s}^{a}, \mathfrak{s}^{b}\right]$.

Для доведення більш загального твердження щодо композицій комутаторів на довільних розміщеннях з повтореннями з підалгебр $\mathfrak{s}^{a}$, для кожного такого розміщення побудуємо аналоги умови (1) і матриць $A, A_{p}, A_{0}$. Для цього структурні сталі потрібно замінити на їх поліноміальні комбінації та врахувати обмеження на індекси, що нумерують базисні елементи залучених підалгебр. Стовпчики у відповідних допоміжних матрицях нумеруємо наборами індексів $\left(i_{1}, \ldots, i_{N}\right)$ у деякому фіксованому ранжуванні, де $N$ - це кількість підалгебр у розміщенні.

Наслідок 1. Припустимо, що алгебра Лі $\mathfrak{g}_{0} \in$ (неперервною чи послідовною) контракцією алгебри Лi $\mathfrak{g}, \mathfrak{g} \rightarrow \mathfrak{g}_{0}$. Тоді для будь-якого повного прапору підпросторів

$$
\{0\}=V^{0} \subset V^{1} \subset V^{2} \subset \cdots \subset V^{n}=V
$$

базового простору $V$ існує такий повний прапор підпросторів

$$
\{0\}=V_{0}^{0} \subset V_{0}^{1} \subset V_{0}^{2} \subset \cdots \subset V_{0}^{n}=V
$$

цього ж простору, що виконуються нерівності $\operatorname{dim}\left[V_{0}^{i}, V_{0}^{j}\right]_{0} \leqslant \operatorname{dim}\left[V^{i}, V^{j}\right]$ для будь-яких $i, j \in\{1, \ldots, n\}$, а також аналогічні нерівності для будь-якої композиції комутаторів на довільному розміщенні з повтореннями з підпросторів $V^{1}, \ldots, V^{n}$.

Доведення. Замкненість підпросторів відносно дужки Лі не є суттєвою у двох останніх абзацах доведення теореми 1.

Простий приклад контракцій до абелевої алгебри показує, що теорему 1 не можна поширити на характеристичні ідеали та мегаідеали. Дійсно, при контракції до абелевої ал- 
гебри центр збільшується до всієї алгебри, але похідна алгебра стискується до нуля. Більш того, в абелевій алгебрі немає власних характеристичних ідеалів та мегаідеалів.

Низку необхідних критеріїв існування контракцій можна отримати як прості наслідки теореми 1. Так, властивості комутативності, нільпотентності, розв'язності та унімодулярності зберігаються при контракціях, а тому алгебра $\mathfrak{s}_{0}^{a}$ успадковує відповідні властивості алгебри $\mathfrak{s}^{a}$.

Наслідок 2. При контракціях алгебр Лі не зменшують розмірності такі структури:

- максимальні абелеві підалгебри,

- максимальні нільпотентні підалгебри,

- максимальні розв’язні підалгебри,

- максимальні абелеві ідеали,

- максимальні нільпотентні ідеали (нільрадикали),

- максимальні розв'язні ідеали (радикали).

Наслідок 3. При контракціях алгебр Лі розмірності похідних алгебр і степенів алгебр не збільшуються.

Зауваження 1. Наведене доведення теореми 1 та доведення подібних тверджень є достатньо елементарними. Їх можна переписати з використанням розкладу Івасави, з якого випливає таке твердження: будь-яку контракцію між алгебрами Лі можна реалізувати трикутними матрицями (див., наприклад, [3]).

Зауваження 2. Теорему 1 і наслідок 1 можна легко узагальнити на довільні скінченновимірні алгебри.

Приклад застосування. Покажемо, що теорему 1 можна ефективно застосовувати для перевірки неіснування контракції в парі фіксованих алгебр у випадках, коли інші критерії такого неіснування не працюють. Для цього необхідно навести класифікації п'яти- і шестивимірних нільпотентних алгебр Лі.

Неізоморфні п’ятивимірні нільпотентні алгебри Лі над $\mathbb{F}=\mathbb{C}$ або $\mathbb{F}=\mathbb{R}$ вичерпують такі алгебри:

$$
\begin{array}{ll}
\mathfrak{g}_{5.6}: & {\left[e_{1}, e_{2}\right]=e_{3},\left[e_{1}, e_{3}\right]=e_{4},\left[e_{1}, e_{4}\right]=e_{5},\left[e_{2}, e_{3}\right]=e_{5} ;} \\
\mathfrak{g}_{5.5}: & {\left[e_{1}, e_{2}\right]=e_{3},\left[e_{1}, e_{3}\right]=e_{4},\left[e_{1}, e_{4}\right]=e_{5} ;} \\
\mathfrak{g}_{5.4}: & {\left[e_{1}, e_{2}\right]=e_{3},\left[e_{1}, e_{3}\right]=e_{4},\left[e_{2}, e_{3}\right]=e_{5} ;} \\
\mathfrak{g}_{5.3}: & {\left[e_{1}, e_{2}\right]=e_{3},\left[e_{1}, e_{4}\right]=e_{5},\left[e_{2}, e_{3}\right]=e_{5} ;} \\
\mathfrak{g}_{5.2}: & {\left[e_{1}, e_{2}\right]=e_{4},\left[e_{1}, e_{3}\right]=e_{5} ;} \\
\mathfrak{g}_{5.1}: & {\left[e_{1}, e_{2}\right]=e_{5},\left[e_{3}, e_{4}\right]=e_{5} ;} \\
\mathfrak{g}_{4} \oplus \mathfrak{g}_{1}: & {\left[e_{1}, e_{2}\right]=e_{3},\left[e_{1}, e_{3}\right]=e_{4} ;} \\
\mathfrak{g}_{3} \oplus 2 \mathfrak{g}_{1}: & {\left[e_{1}, e_{2}\right]=e_{3} ;} \\
5 \mathfrak{g}_{1} . &
\end{array}
$$

У цьому переліку кожну алгебру представлено їі ненульовими комутаційними співвідношеннями з точністю до антисиметричності дужки Лі в базисі $\left(e_{1}, e_{2}, e_{3}, e_{4}, e_{5}\right)$. Алгебри $\mathfrak{g}_{1}$, $\mathfrak{g}_{3}, \mathfrak{g}_{4} \in$ відповідно одновимірною (абелевою) алгеброю, тривимірною (єдиною з точністю до ізоморфізмів нерозкладною нільпотентною) алгеброю Гейзенберга та єдиною з точністю до ізоморфізмів чотиривимірною нерозкладною нільпотентною алгеброю, а $m \mathfrak{g}_{1}$ позначає пряму суму $m$ копій алгебри $\mathfrak{g}_{1}$. 
У [3] доведено, що є такі й лише такі власні контракції між п'ятивимірними нільпотентними алгебрами Лі:

$$
\begin{aligned}
& \mathfrak{g}_{5.6} \rightarrow \mathfrak{g}_{5.5}, \quad \mathfrak{g}_{5.6} \rightarrow \mathfrak{g}_{5.4}, \quad \mathfrak{g}_{5.6} \rightarrow \mathfrak{g}_{5.3}, \quad \mathfrak{g}_{5.6} \rightarrow \mathfrak{g}_{5.2}, \quad \mathfrak{g}_{5.6} \rightarrow \mathfrak{g}_{5.1}, \quad \mathfrak{g}_{5.6} \rightarrow \mathfrak{g}_{4} \oplus \mathfrak{g}_{1}, \\
& \mathfrak{g}_{5.6} \rightarrow \mathfrak{g}_{3} \oplus 2 \mathfrak{g}_{1}, \quad \mathfrak{g}_{5.5} \rightarrow \mathfrak{g}_{5.2}, \quad \mathfrak{g}_{5.5} \rightarrow \mathfrak{g}_{4} \oplus \mathfrak{g}_{1}, \quad \mathfrak{g}_{5.5} \rightarrow \mathfrak{g}_{3} \oplus 2 \mathfrak{g}_{1}, \\
& \mathfrak{g}_{5.4} \rightarrow \mathfrak{g}_{5.2}, \quad \mathfrak{g}_{5.4} \rightarrow \mathfrak{g}_{4} \oplus \mathfrak{g}_{1}, \quad \mathfrak{g}_{5.4} \rightarrow \mathfrak{g}_{3} \oplus 2 \mathfrak{g}_{1}, \\
& \mathfrak{g}_{5.3} \rightarrow \mathfrak{g}_{5.2}, \quad \mathfrak{g}_{5.3} \rightarrow \mathfrak{g}_{5.1}, \quad \mathfrak{g}_{5.3} \rightarrow \mathfrak{g}_{4} \oplus \mathfrak{g}_{1}, \quad \mathfrak{g}_{5.3} \rightarrow \mathfrak{g}_{3} \oplus 2 \mathfrak{g}_{1}, \\
& \mathfrak{g}_{5.2} \rightarrow \mathfrak{g}_{3} \oplus 2 \mathfrak{g}_{1}, \quad \mathfrak{g}_{5.1} \rightarrow \mathfrak{g}_{3} \oplus 2 \mathfrak{g}_{1}, \quad \mathfrak{g}_{4} \oplus \mathfrak{g}_{1} \rightarrow \mathfrak{g}_{5.2}, \quad \mathfrak{g}_{4} \oplus \mathfrak{g}_{1} \rightarrow \mathfrak{g}_{3} \oplus 2 \mathfrak{g}_{1}, \quad * \rightarrow 5 \mathfrak{g}_{1},
\end{aligned}
$$

де остання формула позначає сукупність тривіальних контракцій п'ятивимірних нільпотентних алгебр Лі до п’ятивимірної абелевої алгебри $5 \mathfrak{g}_{1}$.

У літературі існує багато класифікацій шестивимірних нільпотентних алгебр Лі над різними полями. Зокрема, шестивимірні нільпотентні алгебри Лі над $\mathbb{C}$ вперше прокласифікував ще К.А. Умлауф [5], а над довільним полем характеристики 0 - В.В. Морозов [6]. У цій статті використаємо пізнішу класифікацію Л. Магніна [7] шестивимірних нільпотентних алгебр над $\mathbb{C}$ з незначною модифікацією комутаційних співвідношень алгебри $\mathfrak{g}_{5.3} \oplus \mathfrak{g}_{1}$. Отже, неізоморфні шестивимірні комплексні нільпотентні алгебри Лі вичерпують такі алгебри:

$$
\begin{aligned}
& \mathfrak{g}_{6.20}: \quad\left[e_{1}, e_{2}\right]=e_{3},\left[e_{1}, e_{3}\right]=e_{4},\left[e_{1}, e_{4}\right]=e_{5},\left[e_{2}, e_{3}\right]=e_{5},\left[e_{2}, e_{5}\right]=e_{6},\left[e_{3}, e_{4}\right]=-e_{6} ; \\
& \mathfrak{g}_{6.19}: \quad\left[e_{1}, e_{2}\right]=e_{3},\left[e_{1}, e_{3}\right]=e_{4},\left[e_{1}, e_{4}\right]=e_{5},\left[e_{1}, e_{5}\right]=e_{6},\left[e_{2}, e_{3}\right]=e_{5},\left[e_{2}, e_{4}\right]=e_{6} ; \\
& \mathfrak{g}_{6.18}: \quad\left[e_{1}, e_{2}\right]=e_{3},\left[e_{1}, e_{3}\right]=e_{4},\left[e_{1}, e_{4}\right]=e_{5},\left[e_{2}, e_{5}\right]=e_{6},\left[e_{3}, e_{4}\right]=-e_{6} ; \\
& \mathfrak{g}_{6.17}: \quad\left[e_{1}, e_{2}\right]=e_{3},\left[e_{1}, e_{3}\right]=e_{4},\left[e_{1}, e_{4}\right]=e_{5},\left[e_{1}, e_{5}\right]=e_{6},\left[e_{2}, e_{3}\right]=e_{6} ; \\
& \mathfrak{g}_{6.16}: \quad\left[e_{1}, e_{2}\right]=e_{3},\left[e_{1}, e_{3}\right]=e_{4},\left[e_{1}, e_{4}\right]=e_{5},\left[e_{1}, e_{5}\right]=e_{6} ; \\
& \mathfrak{g}_{6.15}: \quad\left[e_{1}, e_{2}\right]=e_{3},\left[e_{1}, e_{3}\right]=e_{4},\left[e_{1}, e_{5}\right]=e_{6},\left[e_{2}, e_{3}\right]=e_{5},\left[e_{2}, e_{4}\right]=e_{6} ; \\
& \mathfrak{g}_{6.14}: \quad\left[e_{1}, e_{2}\right]=e_{3},\left[e_{1}, e_{3}\right]=e_{4},\left[e_{1}, e_{4}\right]=e_{5},\left[e_{2}, e_{3}\right]=e_{6} \text {; } \\
& \mathfrak{g}_{6.13}: \quad\left[e_{1}, e_{2}\right]=e_{4},\left[e_{1}, e_{4}\right]=e_{5},\left[e_{1}, e_{5}\right]=e_{6},\left[e_{2}, e_{3}\right]=e_{5},\left[e_{3}, e_{4}\right]=-e_{6} \text {; } \\
& \mathfrak{g}_{6.12}: \quad\left[e_{1}, e_{2}\right]=e_{4},\left[e_{1}, e_{4}\right]=e_{5},\left[e_{1}, e_{5}\right]=e_{6},\left[e_{2}, e_{3}\right]=e_{6},\left[e_{2}, e_{4}\right]=e_{6} ; \\
& \mathfrak{g}_{6.11}: \quad\left[e_{1}, e_{2}\right]=e_{4},\left[e_{1}, e_{4}\right]=e_{5},\left[e_{1}, e_{5}\right]=e_{6},\left[e_{2}, e_{3}\right]=e_{6} ; \\
& \mathfrak{g}_{6.10}: \quad\left[e_{1}, e_{2}\right]=e_{4},\left[e_{1}, e_{3}\right]=e_{5},\left[e_{1}, e_{4}\right]=e_{6},\left[e_{3}, e_{5}\right]=e_{6} \text {; } \\
& \mathfrak{g}_{6.9}: \quad\left[e_{1}, e_{2}\right]=e_{4},\left[e_{1}, e_{3}\right]=e_{5},\left[e_{2}, e_{5}\right]=e_{6},\left[e_{3}, e_{4}\right]=e_{6} ; \\
& \mathfrak{g}_{6.8}: \quad\left[e_{1}, e_{2}\right]=e_{4},\left[e_{1}, e_{4}\right]=e_{5},\left[e_{2}, e_{3}\right]=e_{5},\left[e_{2}, e_{4}\right]=e_{6} \text {; } \\
& \mathfrak{g}_{6.7}: \quad\left[e_{1}, e_{2}\right]=e_{4},\left[e_{1}, e_{3}\right]=e_{5},\left[e_{1}, e_{4}\right]=e_{6},\left[e_{2}, e_{3}\right]=-e_{6} \text {; } \\
& \mathfrak{g}_{6.6}: \quad\left[e_{1}, e_{2}\right]=e_{4},\left[e_{2}, e_{3}\right]=e_{6},\left[e_{2}, e_{4}\right]=e_{5} \text {; } \\
& \mathfrak{g}_{6.5}: \quad\left[e_{1}, e_{2}\right]=e_{4},\left[e_{1}, e_{4}\right]=e_{5},\left[e_{2}, e_{3}\right]=e_{6},\left[e_{2}, e_{4}\right]=e_{6} \text {; } \\
& \mathfrak{g}_{6.4}: \quad\left[e_{1}, e_{2}\right]=e_{4},\left[e_{1}, e_{3}\right]=e_{6},\left[e_{2}, e_{4}\right]=e_{5} \text {; } \\
& \mathfrak{g}_{6.3}: \quad\left[e_{1}, e_{2}\right]=e_{4},\left[e_{1}, e_{3}\right]=e_{5},\left[e_{2}, e_{3}\right]=e_{6} ; \\
& \mathfrak{g}_{6.2}: \quad\left[e_{1}, e_{2}\right]=e_{5},\left[e_{1}, e_{5}\right]=e_{6},\left[e_{3}, e_{4}\right]=e_{6} ; \\
& \mathfrak{g}_{6.1}: \quad\left[e_{1}, e_{2}\right]=e_{5},\left[e_{1}, e_{4}\right]=e_{6},\left[e_{2}, e_{3}\right]=e_{6} ; \\
& \mathfrak{g}_{5.6} \oplus \mathfrak{g}_{1}:\left[e_{1}, e_{2}\right]=e_{3},\left[e_{1}, e_{3}\right]=e_{4},\left[e_{1}, e_{4}\right]=e_{5},\left[e_{2}, e_{3}\right]=e_{5} ; \\
& \mathfrak{g}_{5.5} \oplus \mathfrak{g}_{1}: \quad\left[e_{1}, e_{2}\right]=e_{3},\left[e_{1}, e_{3}\right]=e_{4},\left[e_{1}, e_{4}\right]=e_{5} ; \\
& \mathfrak{g}_{5.4} \oplus \mathfrak{g}_{1}:\left[e_{1}, e_{2}\right]=e_{3},\left[e_{1}, e_{3}\right]=e_{4},\left[e_{2}, e_{3}\right]=e_{5} ;
\end{aligned}
$$


$\mathfrak{g}_{5.3} \oplus \mathfrak{g}_{1}:\left[e_{1}, e_{2}\right]=e_{3},\left[e_{1}, e_{4}\right]=e_{5},\left[e_{2}, e_{3}\right]=e_{5} ;$

$\mathfrak{g}_{5.2} \oplus \mathfrak{g}_{1}:\left[e_{1}, e_{2}\right]=e_{4},\left[e_{1}, e_{3}\right]=e_{5} ;$

$\mathfrak{g}_{5.1} \oplus \mathfrak{g}_{1}: \quad\left[e_{1}, e_{2}\right]=e_{5},\left[e_{3}, e_{4}\right]=e_{5} ;$

$\mathfrak{g}_{4} \oplus 2 \mathfrak{g}_{1}:\left[e_{1}, e_{2}\right]=e_{3},\left[e_{1}, e_{3}\right]=e_{4} ;$

$\mathfrak{g}_{3} \oplus \mathfrak{g}_{3}: \quad\left[e_{1}, e_{2}\right]=e_{3},\left[e_{4}, e_{5}\right]=e_{6} ;$

$\mathfrak{g}_{3} \oplus 3 \mathfrak{g}_{1}: \quad\left[e_{1}, e_{2}\right]=e_{3}$;

$6 \mathfrak{g}_{1}$.

Згідно з [6], над дійсним полем на додаток до наведених вище існують ще чотири неізоморфні структури:

$\begin{array}{ll}\mathfrak{g}_{6.15}^{\mathbb{R}}: & {\left[e_{1}, e_{2}\right]=e_{3},\left[e_{1}, e_{3}\right]=e_{4},\left[e_{1}, e_{4}\right]=e_{6},\left[e_{2}, e_{3}\right]=e_{5},\left[e_{2}, e_{5}\right]=e_{6} ;} \\ \mathfrak{g}_{6.9}^{\mathbb{R}}: & {\left[e_{1}, e_{3}\right]=e_{4},\left[e_{1}, e_{4}\right]=e_{6},\left[e_{2}, e_{3}\right]=e_{5},\left[e_{2}, e_{5}\right]=e_{6} ;} \\ \mathfrak{g}_{6.5}^{\mathbb{R}}: & {\left[e_{1}, e_{2}\right]=e_{3},\left[e_{1}, e_{3}\right]=e_{5},\left[e_{1}, e_{4}\right]=e_{6},\left[e_{2}, e_{4}\right]=e_{5},\left[e_{2}, e_{3}\right]=-e_{6} ;} \\ \left(2 \mathfrak{g}_{3}\right)^{\mathbb{R}}: & {\left[e_{1}, e_{3}\right]=e_{5},\left[e_{1}, e_{4}\right]=e_{6},\left[e_{2}, e_{4}\right]=e_{5},\left[e_{2}, e_{3}\right]=-e_{6} .}\end{array}$

Тут позначення $\mathfrak{a}^{\mathbb{R}}$ вказує, що комплексифікація дійсної алгебри Лі $\mathfrak{a}^{\mathbb{R}}$ ізоморфна комплексифікації дійсної алгебри Лі $\mathfrak{a}$, хоча самі алгебри $\mathfrak{a}^{\mathbb{R}}$ i $\mathfrak{a}$ неізоморфні.

Лема 2. (i) Не існує контракиій дійсних алгебр $\mathfrak{g}_{6.2}, \mathfrak{g}_{6.4}, \mathfrak{g}_{6.5}, \mathfrak{g}_{6.9}, \mathfrak{g}_{6.9}^{\mathbb{R}}, \mathfrak{g}_{6.10}, \mathfrak{g}_{6.11}, \mathfrak{g}_{6.12}$, $\mathfrak{g}_{6.14}, \mathfrak{g}_{6.17}, \mathfrak{g}_{6.18}$ до алгебри $\left(2 \mathfrak{g}_{3}\right)^{\mathbb{R}}$.

(ii) Не існує контракцій дійсних алгебр $\mathfrak{g}_{6.12}, \mathfrak{g}_{6.14}, \mathfrak{g}_{6.17}, \mathfrak{g}_{6.18}$ до алгебри $\mathfrak{g}_{6.5}^{\mathbb{R}}$.

Доведення. Складність доведення цього твердження полягає в тому, що між відповідними комплексифікаціями наведених алгебр контракції існують. Оскільки стандартні критерії неіснування контракції, зокрема наведені в наслідках 2 і 3, використовують характеристики, які однакові для дійсних алгебр та їх комплексифікацій, вони не працюють у цій ситуації.

Щоб застосувати теорему 1, розглянемо п'ятивимірні підалгебри всіх зазначених алгебр.

Нехай $V=\left\langle e_{1}, e_{2}, e_{3}, e_{4}, e_{5}, e_{6}\right\rangle-$ шестивимірний базовий простір, на якому визначено всі ці алгебри. П'ятивимірні підпростори простору $V$ можна розбити на такі сім'ї:

$$
\begin{aligned}
& \mathcal{F}_{1}=\left\{\left\langle e_{2}, e_{3}, e_{4}, e_{5}, e_{6}\right\rangle\right\}, \\
& \mathcal{F}_{2}=\left\{\left\langle e_{1}+\alpha e_{2}, e_{3}, e_{4}, e_{5}, e_{6}\right\rangle \mid \alpha \in \mathbb{R}\right\}, \\
& \mathcal{F}_{3}=\left\{\left\langle e_{1}+\alpha e_{3}, e_{2}+\beta e_{3}, e_{4}, e_{5}, e_{6}\right\rangle \mid \alpha, \beta \in \mathbb{R}\right\}, \\
& \mathcal{F}_{4}=\left\{\left\langle e_{1}+\alpha e_{4}, e_{2}+\beta e_{4}, e_{3}+\gamma e_{4}, e_{5}, e_{6}\right\rangle \mid \alpha, \beta, \gamma \in \mathbb{R}\right\}, \\
& \mathcal{F}_{5}=\left\{\left\langle e_{1}+\alpha e_{5}, e_{2}+\beta e_{5}, e_{3}+\gamma e_{5}, e_{4}+\delta e_{5}, e_{6}\right\rangle \mid \alpha, \beta, \gamma, \delta \in \mathbb{R}\right\}, \\
& \mathcal{F}_{6}=\left\{\left\langle e_{1}+\alpha e_{6}, e_{2}+\beta e_{6}, e_{3}+\gamma e_{6}, e_{4}+\delta e_{6}, e_{5}+\varepsilon e_{6}\right\rangle \mid \alpha, \beta, \gamma, \delta, \varepsilon \in \mathbb{R}\right\} .
\end{aligned}
$$

Доведемо, що всі п'ятивимірні підалгебри алгебри $\left(2 \mathfrak{g}_{3}\right)^{\mathbb{R}}$ ізоморфні алгебрі $\mathfrak{g}_{5.2} .3$ наведеної класифікації п’ятивимірних нільпотентних алгебр Лі легко бачити, що характеристичними властивостями алгебри $\mathfrak{g}=\mathfrak{g}_{5.2}$ є дві рівності: $\operatorname{dim} \mathfrak{g}^{2}=2$ i $\operatorname{dim} \mathfrak{g}^{3}=0$, де $\mathfrak{g}^{k}$ позначає $k$-й степінь алгебри $\mathfrak{g}$. Друга рівність виконується для будь-якої підалгебри алгебри $\mathfrak{g}_{5.2}$, бо вона виконується для самої алгебри. Оскільки $\left[e_{2}+\beta e_{5}, e_{4}+\gamma e_{5}\right]=e_{5}$ i $\left[e_{1}+\alpha e_{6}, e_{4}+\gamma e_{6}\right]=e_{6}$, то підпростори з сімей $\mathcal{F}_{5}$ і $\mathcal{F}_{6}$ не замкнені відносно дужки Лі, а тому 
не є підалгебрами. Підпростори, що містять похідну $\left(\left(2 \mathfrak{g}_{3}\right)^{\mathbb{R}}\right)^{\prime}$, тобто всі підпростори 3 сімей $\mathcal{F}_{1}-\mathcal{F}_{4}$, є підалгебрами (навіть ідеалами) в $\left(2 \mathfrak{g}_{3}\right)^{\mathbb{R}}$. Прокомутувавши базисні елементи кожної з цих підалгебр, отримаємо, що їх похідні збігаються з $\left\langle e_{5}, e_{6}\right\rangle$, тобто вони ізоморфні алгебрі $\mathfrak{g}_{5.2}=-1$.

Аналогічно покажемо, що всі п’ятивимірні підалгебри алгебри $\mathfrak{g}_{6.5}^{\mathbb{R}}$ ізоморфні або алгебрі $\mathfrak{g}_{5.2}$, або алгебрі $\mathfrak{g}_{5.4}$. Підпростори з сімей $\mathcal{F}_{3}, \mathcal{F}_{5}, \mathcal{F}_{6}$ та з сім'ї $\mathcal{F}_{4} 3 \gamma \neq 0$ не замкнені відносно дужки Лі, оскільки $\left[e_{1}+\alpha e_{3}, e_{2}+\beta e_{3}\right]=e_{3}+\beta e_{5}+\alpha e_{6},\left[e_{1}+\alpha e_{5}, e_{3}+\gamma e_{5}\right]=e_{5},\left[e_{1}+\alpha e_{6}, e_{4}+\delta e_{6}\right]=e_{6}$, $\left[e_{1}+\alpha e_{4}, e_{2}+\beta e_{4}\right]=e_{3}+\beta e_{6}-\alpha e_{5}$. Усі інші підпростори є підалгебрами i, більш того, ідеалами в $\mathfrak{g}_{6.5}^{\mathbb{R}}$, причому підпростори 3 сімей $\mathcal{F}_{1}, \mathcal{F}_{4}$ знову ізоморфні алгебрі $\mathfrak{g}_{5.2}$, а підпростори 3 сім’ї $\mathcal{F}_{4}$ з $\gamma=0$ ізоморфні алгебрі $\mathfrak{g}_{5.4}$. Стандартні комутаційні співвідношення алгебри $\mathfrak{g}_{5.4}$ отримаємо в базисі $\tilde{e}_{1}=e_{1}+\alpha e_{4}, \tilde{e}_{2}=e_{2}+\beta e_{4}, \tilde{e}_{3}=e_{3}-\alpha e_{5}+\beta e_{6}, \tilde{e}_{4}=e_{5}, \tilde{e}_{5}=-e_{6}$.

Щоб довести відсутність контракцій у зазначених у лемі парах алгебр, достатньо вказати в кожній з початкових алгебр підалгебру, що не контрактує до алгебри $\mathfrak{g}_{5.2}$, а отже, і до алгебри $\mathfrak{g}_{5.4}$. Така підалгебра має бути ізоморфною одній з алгебр $\mathfrak{g}_{5.1}, \mathfrak{g}_{3} \oplus 2 \mathfrak{g}_{1}, 5 \mathfrak{g}_{1}$. Лінійні оболонки $\left\langle e_{2}, e_{3}, e_{4}, e_{5}, e_{6}\right\rangle$ i $\left\langle e_{1}, e_{3}, e_{4}, e_{5}, e_{6}\right\rangle$ відповідно в кожній з алгебр $\mathfrak{g}_{6.17}, \mathfrak{g}_{6.11}$, $\mathfrak{g}_{6.14}, \mathfrak{g}_{6.12}, \mathfrak{g}_{6.10}, \mathfrak{g}_{6.4}, \mathfrak{g}_{6.2}$ і в алгебрі $\mathfrak{g}_{6.5} \in$ підалгебрами, ізоморфними алгебрі $\mathfrak{g}_{3} \oplus 2 \mathfrak{g}_{1}$, а лінійні оболонки $\left\langle e_{2}, e_{3}, e_{4}, e_{5}, e_{6}\right\rangle \mathrm{i}\left\langle e_{1}, e_{2}, e_{4}, e_{5}, e_{6}\right\rangle$ відповідно в кожній з алгебр $\mathfrak{g}_{6.9}, \mathfrak{g}_{6.18} \mathrm{i}$ в алгебрі $\mathfrak{g}_{6.9}^{\mathbb{R}} \in$ підалгебрами, ізоморфними алгебрі $\mathfrak{g}_{5.1}$, що завершує доведення леми.

Зауваження 3.3 доведення леми 2 очевидно, що не існує також контракцій алгебр $\mathfrak{g}_{6.2}$, $\mathfrak{g}_{6.4}, \mathfrak{g}_{6.5}, \mathfrak{g}_{6.9}, \mathfrak{g}_{6.9}^{\mathbb{R}}, \mathfrak{g}_{6.10}, \mathfrak{g}_{6.11}, \mathfrak{g}_{6.12}, \mathfrak{g}_{6.14}$ до алгебри $\mathfrak{g}_{6.5}^{\mathbb{R}}$. Водночас, ці алгебри відрізняються від наведених у пункті (ii) леми 2 тим, що контракцій не існує і між відповідними комплексифікаціями, а їх відсутність можна довести з використанням стандартних критеріїв, які однаково працюють у дійсному і комплексному випадках, як-то розмірності алгебри диференціювань, похідної чи центру [8].

Зауваження 4. Як випливає з розгляду в [9], а також неопублікованих результатів М.О. Нестеренко та Р.О. Поповича, алгебра $\mathfrak{g}_{6.18}$ контрактує до алгебр $\mathfrak{g}_{6.2}, \mathfrak{g}_{6.4}, \mathfrak{g}_{6.5}$, $\mathfrak{g}_{6.9}, \mathfrak{g}_{6.9}^{\mathbb{R}}, \mathfrak{g}_{6.10}, \mathfrak{g}_{6.11}, \mathfrak{g}_{6.12}, \mathfrak{g}_{6.14}$. Тому в лемі 2 достатньо довести відсутність контракцій $\mathfrak{g}_{6.18}$ До $\mathfrak{g}_{6.5}^{\mathbb{R}}$ i $\left(2 \mathfrak{g}_{3}\right)^{\mathbb{R}}$.

\section{ЦИТОВАНА ЛІТЕРАТУРА.}

1. Burde D. Degenerations of nilpotent Lie algebras. J. Lie Theory. 1999. 9, № 1. P. 193-202.

2. Gorbatsevich V.V., Onishchik A.L., Vinberg E.B. Lie groups and Lie algebras. III. Structure of Lie groups and Lie algebras. Berlin: Springer, 1997. xx+328 pp.

3. Grunewald F., O'Halloran J. Varieties of nilpotent Lie algebras of dimension less than six. J. Algebra. 1988. 112, № 2. P. 315-325. https://doi.org/10.1016/0021-8693(88)90093-2

4. Popovych D.R. Contractions with necessarily unbounded matrices. Linear Algebra Appl. 2014. 458. P. 689698. https://doi.org/10.1016/j.laa.2014.06.017

5. Umlauf K.A. Über die Zusammensetzung der endlichen continuierlichen Transformationsgruppen, insbesondere der Gruppen von Range Null: Ph.D. thesis / University of Leipzig, 1891.

6. Морозов В.В. Классификация нильпотентных алгебр Ли шестого порядка. Изв. вузов. Матем. 1958. № 4. C. $161-171$.

7. Magnin L. Sur les algebres de Lie nilpotentes de dimension \$7. J. Geom. Phys. 1986. 3, № 1. P. 119-144. https://doi.org/10.1016/0393-0440(86)90005-7

8. Nesterenko M., Popovych R.O. Contractions of low-dimensional Lie algebras. J. Math. Phys. 2006. 47, № 12. 123515. https://doi.org/10.1063/1.2400834 
9. Seeley C. Degenerations of 6-dimensional nilpotent Lie algebras over $\mathbb{C}$. Arch. Math. 1990. 56, № 10. P. 236-241. https://doi.org/10.1080/00927879008824088

Надійшло до редакції 29.06.2021

\section{REFERENCES}

1. Burde, D. (1999). Degenerations of nilpotent Lie algebras. J. Lie Theory, 9, No. 1, pp. 193-202.

2. Gorbatsevich, V. V., Onishchik, A. L. \& Vinberg, E. B. (1997). Lie groups and Lie algebras. III. Structure of Lie groups and Lie algebras. Berlin: Springer.

3. Grunewald, F. \& O'Halloran, J. (1988). Varieties of nilpotent Lie algebras of dimension less than six. J. Algebra, 112, No. 2, pp. 315-325. https://doi.org/10.1016/0021-8693(88)90093-2

4. Popovych, D. R. (2014). Contractions with necessarily unbounded matrices. Linear Algebra Appl., 458, pp. 689-698. https://doi.org/10.1016/j.laa.2014.06.017

5. Umlauf, K. A. (1891). Über die Zusammensetzung der endlichen continuierlichen Transformationsgruppen, insbesondere der Gruppen von Range Null. (Unpublished Ph.D. thesis). University of Leipzig, Leipzig, Germany.

6. Morozov, V. V. (1958). Classification of nilpotent Lie algebras of sixth order. Izvestiya VUZ. Matematika, No. 4, pp. 161-171 (in Russian).

7. Magnin, L. (1986). Sur les algebres de Lie nilpotentes de dimension $\leqslant 7$. J. Geom. Phys., 3, No. 1, pp. 119-144. https://doi.org/10.1016/0393-0440(86)90005-7

8. Nesterenko, M. \& Popovych, R. O. (2006). Contractions of low-dimensional Lie algebras. J. Math. Phys., 47, No. 12, 123515. https://doi.org/10.1063/1.2400834

9. Seeley, C. (1990). Degenerations of 6-dimensional nilpotent Lie algebras over $\mathbb{C}$. Arch. Math., 56, No. 10, pp. 236-241. https://doi.org/10.1080/00927879008824088

Received 29.06.2021

\section{R. Popozych}

Institute of Mathematics of the NAS of Ukraine, Kyiv

E-mail: deviuss@gmail.com

\section{FLAGS OF SUBALGEBRAS IN CONTRACTED LIE ALGEBRAS}

We prove a theorem that describes the behavior of subalgebra flags of Lie algebras under contractions and can be applied as a new criterion for the non-existence of contractions. A weaker version of the theorem is obtained for flags of subspaces. Using the theorem, we prove the non-existence of contractions for a number of pairs of sixdimensional nilpotent real Lie algebras, for which the earlier known criteria do not work.

Keywords: contractions of Lie algebras, flags of subalgebras, flags of subspaces in Lie algebras, six-dimensional nilpotent Lie algebras. 